\title{
A proteomic evaluation of urinary changes associated with cardiopulmonary bypass
}

Ravi C. Dwivedi1,2, Mario Navarrete ${ }^{1,2}$, Nora Choi ${ }^{1,2,5}$, Victor Spicer $^{1,2}$, Claudio Rigatto $^{3}$, Rakesh C. Arora ${ }^{4,5}$, Oleg Krokhin ${ }^{1,2}$, Julie Ho ${ }^{1,2,3,6}$ and John A. Wilkins ${ }^{1,2^{*}}$

\begin{abstract}
Background: The urinary proteome of patients undergoing cardiopulmonary bypass (CPB) may provide important insights into systemic and renal changes associated with the procedure. Such information may ultimately provide a basis to differentiate changes or properties associated with the development of acute kidney injury. While mass spectrometry (MS) analysis offers the potential for in-depth compositional analysis it is often limited in coverage and relative quantitation capacity. The aim of this study was to develop a process flow for the preparation and comparison of the intraoperative urinary proteome.
\end{abstract}

Methods: Urines were collected from patients at the start of CPB and 1-h into CPB. Pooled samples $(n=5)$ from each time point were processed using a modified Filter Assisted Sample Preparation protocol. The resulting peptides were analyzed by 2D-LC-MS/MS and by 1D-LC-MS/MS SWATH (Sequential Window acquisition of All Theoretical fragment ion spectra).

Results: The 2D-LC-MS/MS analysis identified 1324 proteins in the two pools, of which 744 were quantifiable. The SWATH approach provided quantitation for 730 proteins, 552 of which overlapped with the common population from the 2D-IDA results. Intensity correlation filtering between the two methods gave 475 proteins for biological interpretation. Proteins displaying greater than threefold changes $\left(>\log _{2} 1.59\right)$ at 1-hour CPB relative to the initiation of CPB (26 down-regulated and 22 up-regulated) were selected for further analysis. Up-regulated proteins were enriched in $\mathrm{GO}$ terms related to humoral immune response, predominantly innate immunity ( $\mathrm{C} 4 \mathrm{~b}$, lactotransferrin, protein S100-A8, cathelicidin, myeloperoxidase) and extracellular matrix reorganization (e.g. MMP-9).

Conclusions: This study describes a scheme for processing urine from patients undergoing CPB for mass spectrometry-based analysis. The introduction of SWATH into the workflow offers a sample and instrument sparing approach to obtaining consistent in-depth sample analysis. The design of the methodology is such that it can be readily applied to large numbers of clinical samples with the potential for automation. The results also suggest that activation of the innate immune responses occur during cardiac bypass surgery.

Keywords: Data dependent acquisition (DDA), Information dependent data acquisition (IDA), Data independent acquisition (DIA), Molecular weight cut off (MWCO) filters, Label free quantitation, SWATH, Proteomics, Cardiac surgery, Urine, Renal

\footnotetext{
*Correspondence: John.Wilkins@umanitoba.ca

${ }^{1}$ Manitoba Centre for Proteomics and Systems Biology, University of Manitoba and Health Sciences Centre, Room 799, John Buhler Research Center, 715 Mc Dermot Avenue, Winnipeg, MB R3E 3P4, Canada

Full list of author information is available at the end of the article
} 


\section{Background}

Urine proteomic analysis is a powerful tool that may provide insights into protein changes that are predictive of disease development and to define underlying pathophysiologic processes. Urine provides a noninvasive, rich source of proteins that are potentially informative of systemic and renal processes. Indeed while there are unique aspects to the urine proteome, it has been shown to have approximately $61 \%$ overlap with both the kidney and plasma proteomes [1]. These considerations have been the rationale for a number of proteomic studies of urinary proteins [2-5]. However, urine proteomic analysis is complicated by protein concentration variability, the presence of interfering salts, metabolites and other substances [6]. These considerations often require significant sample processing which can impact the reproducibility of quantitative analysis of urine [7].

Different urine proteomics approaches have been applied to clinical scenarios in the intensive care unit [5, $8]$, renal transplants $[9,10]$, and pre/post-operative cardiac surgery [2]. However, proteomic evaluation of intraoperative urines is particularly challenging because of the presence of unique inhibitory materials that interfere with chromatography. Only targeted SELDI TOF-MS analysis has been successfully applied to intra-operative cardiac surgery urine samples [3, 11, 12], however this approach offers extremely limited proteome coverage and does not provide specific protein identification.

Shotgun proteomics on complex biological samples using in-depth 2D-LC-MS/MS analysis provides maximum proteome coverage and protein identification. However, the stochastic nature of DDA/IDA peptide selection for analysis often results in variable identification coverage between analyses $[1,13,14]$. Sequential Window acquisition of All Theoretical fragment ion spectra Mass-Spectrometry (SWATH-MS) has been proposed as an alternative approach [15]. SWATH-MS offers accurate and reproducible protein quantification from complex samples using data-independent acquisition. A significant advantage of SWATH analysis is that it reports the relative abundance of selected proteins in different samples. SWATH-MS also provides a permanent digital file representing the continuous MS-measurable proteome of a sample that can subsequently be re-analyzed as new approaches and insights become available [16].

This study describes a workflow for the processing and analysis of urines from adult patients undergoing cardiac bypass surgery. A comparison of DDA/IDA and SWATHbased analysis is provided suggesting that the latter may represent a useful adjunct for the comparison of clinical samples.

\section{Methods}

Patients and samples

The study protocol was approved by the Health Research Ethics Board, University of Manitoba and all patients provided informed consent. Patients with stable renal function $(n=5)$ were selected for analysis from a larger prospective observational cohort of adult cardiac surgery patients using the following criteria: baseline estimated glomerular filtration rate (eGFR) $\geq 60 \mathrm{~mL} / \mathrm{min}$ and post-operative serum creatinine rise $<10 \%$ from baseline. Serial urine samples were collected at the initiation of $\mathrm{CPB}$ and after 1 -h on CPB. Urines were spun at $870 \mathrm{~g}$ for $6 \mathrm{~min}$, and the supernatants stored at $-80{ }^{\circ} \mathrm{C}$ for further analysis. The baseline clinical characteristics of the patient cohort are described in Table 1.

\section{Sample preparation for mass-spectrometry analysis}

Total urine protein was quantified using a total Protein Kit, Micro Pyrogallol Red Method (Sigma, USA). A pool of urines from 5 patients ( $40 \mu \mathrm{g}$ protein/patient) was prepared for each time point. The pooled samples were processed using a modified FASP protocol [17]. Pooled urines were treated for $5 \mathrm{~min}$ with $0.1 \mathrm{M} \mathrm{DTT}$ at $100{ }^{\circ} \mathrm{C}$. The cooled samples were brought to $8 \mathrm{M}$ urea, $100 \mathrm{mM}$ Tris $\mathrm{pH} 8.0$

\section{Table 1 Baseline patient characteristics}

\begin{tabular}{|c|c|}
\hline Variable & Patients $(n=5)$ \\
\hline Age (years) & $75(73-77)$ \\
\hline Male & $3(60 \%)$ \\
\hline $\mathrm{eGFR}\left(\mathrm{mL} / \mathrm{min} / 1.72 \mathrm{~m}^{2}\right)$ & $72.5(69.2-76.5)$ \\
\hline Baseline creatinine (mg/dL) & $94(69-97)$ \\
\hline THAKAR Score & $2(1-2)$ \\
\hline Diabetes mellitus & $1(20 \%)$ \\
\hline Chronic obstructive pulmonary disease & $1(20 \%)$ \\
\hline Hospitalized congestive heart failure & $1(20 \%)$ \\
\hline Previous myocardial infarction & $2(40 \%)$ \\
\hline Previous CABG & $0(0 \%)$ \\
\hline Peripheral arterial disease & $1(20 \%)$ \\
\hline Amputation or peripheral arterial disease bypass & $0(0 \%)$ \\
\hline Previous cerebrovascular accident & $0(0 \%)$ \\
\hline Previous transient ischemic attack & $1(20 \%)$ \\
\hline Type of surgery (isolated CABG) & $3(60 \%)$ \\
\hline Pump time (min) & $68(64-95)$ \\
\hline Cross-clamp time (min) & $54(36-78)$ \\
\hline Operating room duration (min) & $254(250-305)$ \\
\hline $\begin{array}{l}\text { European system for cardiac operation risk evaluation } \\
\text { EuroSCORE II }\end{array}$ & $1.5 \%(1.0-2.4 \%)$ \\
\hline Intraoperative urine output (mL) & $1180(800-1350)$ \\
\hline
\end{tabular}

Values expressed as median (interquartile range) or $\mathrm{N}$ (percent). Continuous variables compared using Mann-Whitney test, categorical variables compared using Chi-square or Fisher's Exact Test 
and diluted with an equal volume of the same solution. The samples were transferred to the indicated Amicon MWCO ultra-15 centrifugal filter (Millipore) (i.e. 3, 10 or $30 \mathrm{kDa}$ cut-off) and centrifuged at $4000 \times g$ for $15 \mathrm{~min}$. The retentate was then diluted to $15 \mathrm{ml}$ with $8 \mathrm{M}$ urea, $100 \mathrm{mM}$ Tris $\mathrm{pH} 8.0$ and centrifuged for $30-50 \mathrm{~min}$ at $4000 \times g$ to remove excess DTT. This was repeated once after which $2 \mathrm{ml}$ of $50 \mathrm{mM}$ iodoacetamide was added to the sample and incubated in the dark for $15 \mathrm{~min}$. The sample was centrifuged for $15 \mathrm{~min}$ at $4000 \times g$ and $2 \mathrm{ml}$ of $20 \mathrm{mM}$ DTT was added to react with residual iodoacetamide. The sample was centrifuged for $15 \mathrm{~min}$ at $4000 \times \mathrm{g}$, after which the retentate was brought to $500 \mu \mathrm{l}$ with $50 \mathrm{mM}$ ammonium bicarbonate. Trypsin was added at 1:50 ratio based on the starting protein amount and the sample was incubated overnight at $28{ }^{\circ} \mathrm{C}$ with shaking. The digest was brought to $500 \mathrm{mM}$ $\mathrm{NaCl}$ and gently mixed. The samples were centrifuged for $15 \mathrm{~min}$ at $4000 \times g$. The MWCO filter was washed with $200 \mu \mathrm{l}$ of water by centrifugation for $15 \mathrm{~min}$ at $4000 \times g$. The peptides were eluted from the membrane by centrifugation $(3000 \times g, 15 \mathrm{~min})$ with $500 \mu \mathrm{l}$ of $15 \%$ acetonitrile, $0.1 \%$ trifluoroactic acid in water. The eluted peptides were dried using a speedvac to remove the organic solvents used for elution from the MWCO membranes. The sample was dissolved in $500 \mu \mathrm{l}$ of $0.5 \%$ trifluoroacetic acid and desalted with a C18-SD extraction disc cartridge (Sigma, USA). The eluted peptides were dried and reconstituted in $40 \mu \mathrm{l}$ of $0.1 \%$ formic acid in water. An estimation of peptide quantity was made using a Nano-drop spectrophotometer (Thermo Scientific Nanodrop 2000) at $205 \mathrm{~nm}$ absorbance where 1.0 absorbance unit corresponded to a peptide concentration of $\sim 1 \mu \mathrm{g} / \mu \mathrm{l}$.

\section{D-HPLC-MS/MS data acquisition and peptide identification}

The processed trypsin digested peptides were separated using two-dimensional liquid chromatographic method [18]. C-18 cleaned peptides obtained after modified FASP procedure were gradient fractionated on a C18 X-Terra column, $(1 \times 100 \mathrm{~mm}, 3 \mu \mathrm{m}, 100 \AA$, Waters, Milford, MA). Both eluents A (water) and B (90\% acetonitrile) contained $20 \mathrm{mM}$ ammonium formate buffer $\mathrm{pH} 10.0$. A total of 30 fractions were collected using a gradient of $1-44 \%$ of solvent B in $30 \mathrm{~min}$, at a flow rate of $300 \mu \mathrm{l} /$ min. The resulting fractions were concatenated to eight fractions, dried and dissolved in $50 \mu \mathrm{l}$ of eluent $\mathrm{A}(0.1 \%$ Formic Acid). One microgram from each concatenated fraction was injected into a splitless nano-flow Tempo LC system (Eksigent, Dublin, CA) via a PepMap100 trap column $(0.3 \times 5 \mathrm{~mm}, 5 \mu \mathrm{m}, 100 \AA$ A Dionex, Sunnyvale, CA) and a $100 \mu \mathrm{m} \times 150 \mathrm{~mm}$ analytical column packed with $3 \mu \mathrm{m}$ Luna C18(2) (Phenomenex, Torrance, CA). Both eluents A (2\% acetonitrile in water) and B (98\% acetonitrile) contained $0.1 \%$ formic acid as ion-pairing modifier. A $0.44 \%$ acetonitrile per minute linear gradient $(0-35 \% \mathrm{~B}$ in $80 \mathrm{~min}, 500 \mathrm{nl} / \mathrm{min})$ was used for peptide elution, followed by 5 min wash with $80 \% \mathrm{~B}$.

Samples were analyzed on a 5600 TripleTOF mass spectrometer (SCIEX, Canada) in standard MS/MS data dependent acquisition (DDA) mode also referred as information dependent acquisition (IDA). Each DDA/ IDA cycle included a $250 \mathrm{~ms}$ MS scan $(400-1600 \mathrm{~m} / \mathrm{z})$ and up to $20 \mathrm{MS} / \mathrm{MS}$ (100 ms each, $100-1600 \mathrm{~m} / \mathrm{z}$ ) for ions state of +2 to +5 and an intensity of minimum $300 \mathrm{cps}$. Selected ions and their isotopes were dynamically excluded from further fragmentation for $12 \mathrm{~s}$.

For each pooled sample the resulting eight instrumentlevel WIFF files were converted to MGF format using the conversion tool bundled with Analyst. These were then sequentially concatenated into a single MGF file for each sample pool. Peptide identification and quantitation was done using X!tandem (cyclone 2012.10.01.1) [19]. Peptides were identified against a human database of the June 2015 release of SwissProt with the following search settings: constant modification $\mathrm{C}+57.021$ (cysteine protection); variable modifications: $\mathrm{M}, \mathrm{W}+15.995$ (oxidation) or +31.989 (double oxidation); S, T, Y +79.966 (phosphorylation), N, Q +0.984 (deamidation); parent mass error: \pm 20 PPM, fragment mass error: 0.1 Da. Peptide identification $\log _{10}$ expectation values were computed using a survival function approach $[20,21]$.

\section{SWATH-MS acquisition}

SWATH-MS, also referred to as data independent acquisition (DIA), runs were conducted using a 5600 TripleTOF mass-spectrometer, which operated in a looped product ion mode. This mode helps instrument to allow quadrupole resolution of $25 \mathrm{amu}$ per mass selection. Precursor selection windows had an overlap of $1 \mathrm{Da}$ with their adjacent selection window to ensure complete isotope coverage between SWATH blocks. Each cycle of SWATH analysis consisted of a $100 \mathrm{~ms}$ MS scan and a $100 \mathrm{~ms}$ MS/MS scan in $25 \mathrm{~m} / \mathrm{z}$ blocks in $400-1250 \mathrm{~m} / \mathrm{z}$ range, and a total of 34 SWATH blocks were collected for each scan. An accumulation time of $100 \mathrm{~ms}$ was used for each fragment ion scan and for the survey scans acquired at the beginning of each cycle, resulting in a total cycle time of $3.5 \mathrm{~s}$. The collision energy for each window was determined on the basis of the collision energy for a $2+$ ion centered in the respective window (equation: $0.0625 \times \mathrm{m} / \mathrm{z}-3.5$ ) with a collision energy spread of $15 \mathrm{eV}$. The mass spectrometer was always operated in high sensitivity mode. Peptides were separated using the same LC system configuration as for the second dimension DDA/IDA acquisition, using a $0.30 \%$ acetonitrile per minute linear gradient $(0-33 \% \mathrm{~B}$ in $110 \mathrm{~min}$, $500 \mathrm{nl} / \mathrm{min}$ ), followed by a $5 \mathrm{~min}$ wash with $80 \% \mathrm{~B}$. 


\section{SWATH library and analysis}

For SWATH library construction all non-modified peptides across a large dataset 2D DDA/IDA runs were concatenated into a non-redundant collection using methods described earlier [22]. We opted to include all peptides from a broader study of eight urine pool samples, regardless of their expectation values or number of peptides per protein detected in each run, to yield maximum SWATH depth. The peptide entries from each DDA/IDA run were retention-aligned against a system average value using the retention times extracted for the custom peptides spiked into each sample.

To enhance our potential SWATH extraction coverage, we developed a method to supplement this library with transitions from the comprehensive human SWATH library [23]. These "10K-library" transitions represented peptide fragment ion patterns detected in SWATH runs, which differ somewhat from their DDA/IDA counterparts due to differences in instrument fragmentation settings across the acquisition methods. This library also provided transition versions for multiple potential charge states of a given peptide, a feature that varies between 1D and $2 \mathrm{D}$ runs as a product of finite instrument acquisition time per MS/MS cycle.

This combined library was retention aligned against average retention values acquired from prior DDA/ IDA runs under identical chromatographic settings. This library drove the SWATH analysis component of Peakview (1.2.0.4; AB Sciex 2012). The SWATH parameters were a $6 \mathrm{~min}$ extraction window $( \pm 3 \mathrm{~min})$, a mass accuracy $50 \mathrm{PPM}$, a search depth of 100 peptides per protein, 6 transitions per peptide, and a $1 \%$ false discovery rate. Extraction was performed on all eight SWATH runs of our larger study simultaneously to permit optimal "gap filling", yielding a modest transition detection rate of $\sim 11 \%$ (21,205 of 193,112 targeted transitions) spanning 6050 peptides.

The utility of the custom-peptide [24] based retention alignments is illustrated in (Additional file 1: Table S1). The differences between the library target retention times and their actual extracted retention times in both of our SWATH runs are well within our SWATH retention extraction time window with no apparent systematic skewing of the retention differences, indicating good control over the chromatography across the SWATH runs and between the methods.

\section{Results and discussion}

\section{Optimization of urine sample preparation for mass spectrometry}

The aim of the present study was to develop methods for the reliable comparison of the urinary proteome during $\mathrm{CPB}$. Preliminary studies indicated that urine samples collected during cardiac surgery contained substance(s) that interfered with processing and chromatographic separations of the peptides derived from these samples. However, samples collected post operatively from the same patient group did not appear to contain the chromatographic-interfering substances. Although unproven it was felt that the most likely cause of these interfering effects were due to lipophilic substances possibly propofol, an anaesthetic used during the surgery.

After examining several approaches we established that a modified version of the filter-aided sample preparation (FASP) procedure [17] provided a suitable approach. This procedure involves sample processing with subsequent concentration of the sample using a molecular weight cut off filter. The concentrated sample is then processed in situ and the resulting peptides are eluted for analysis. This approach allows for sample concentration and clean-up in a single reaction vessel, limiting protein loss and minimizing technical variability. The SDS and urea used in this procedure also offered a means of removing hydrophobic interfering substances which were present in the samples collected from patients during surgery. Preliminary experiments determined that samples prepared with either 3 or $10 \mathrm{kDa}$ cut off filters proposed in the original FASP method [17] still contained compounds that altered the chromatographic properties of the recovered peptides (Fig. 1a). The retention times of peptides isolated under these conditions were reduced and there was considerable peak spreading which resulted in suboptimal separations. Increasing the pore size of the filters to $30 \mathrm{kDa}$ molecular weight cut off membrane eliminated this effect suggesting that the interfering materials were removed under these conditions (Fig. 1a). However, there was concern that the increased pore size could also result in the selective loss of lower molecular weight species in the urine samples. A comparative analysis of the $30 \mathrm{kDa}$ membrane processed samples with those produced with 3 or $10 \mathrm{kDa}$ membranes indicated that the $30 \mathrm{kDa}$ sample provided a greater number of proteins in the 1-30 kDa range and overall more protein identifications than the other samples (Fig. 1b). While these results could not exclude the possibility of some protein loss using the $30 \mathrm{kDa}$ molecular weight cut off membranes, it was apparent that the recovery and range of proteins was better with these membranes than with either the 3 or $10 \mathrm{kDa}$ membranes. This increased recovery was demonstrated by the higher numbers of protein identifications and the numbers of proteins with molecular weights less than $30 \mathrm{kDa}$ in samples processed using the $30 \mathrm{kDa}$ MWCO filters (502 and 162, respectively) as compared with the $3 \mathrm{kDa}$ (290 and 97) or $10 \mathrm{kDa}$ (394 and 123) MWCO processed samples. The recent results of Berger et al. suggest that perhaps even larger pore PVDF based 

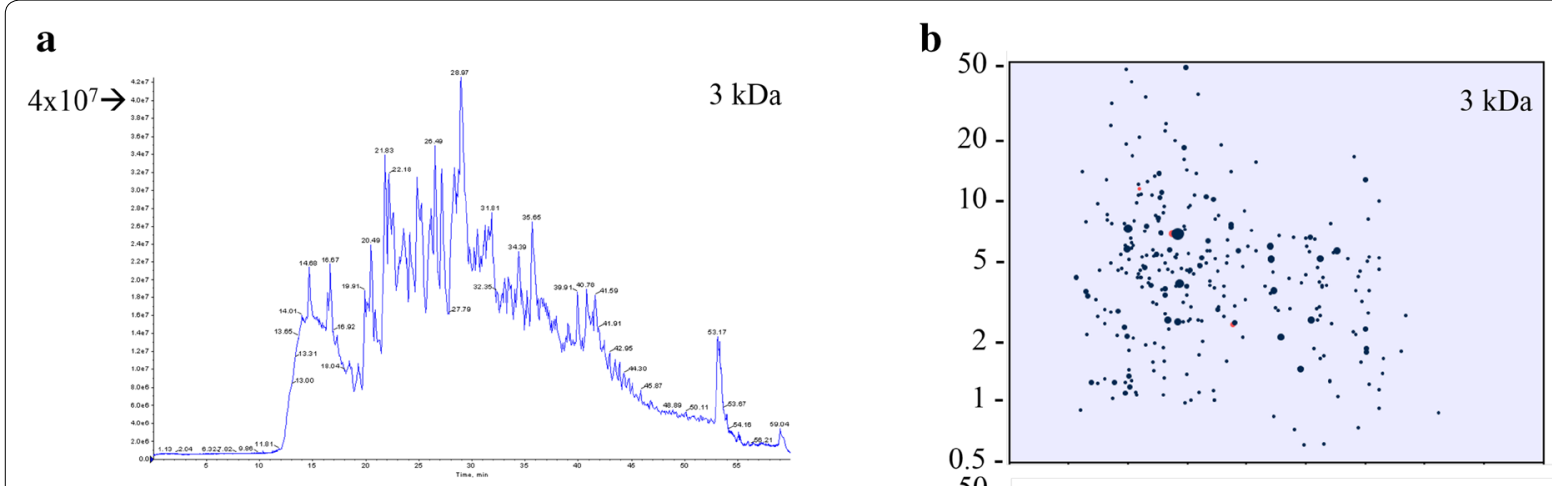

$97 / 290$
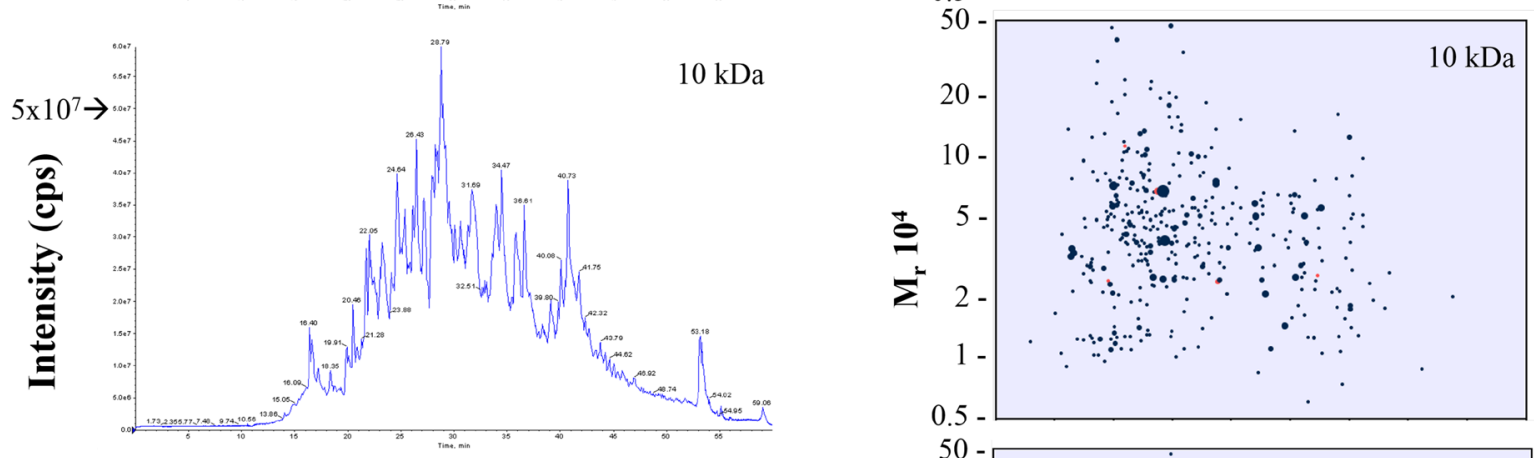

$123 / 394$

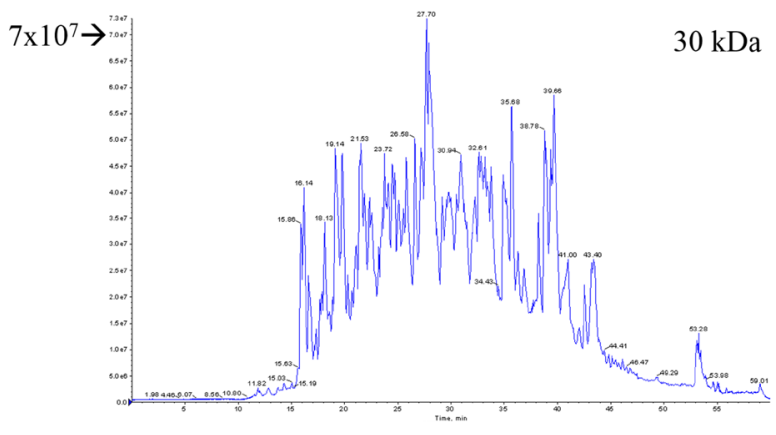

Time (Minutes)

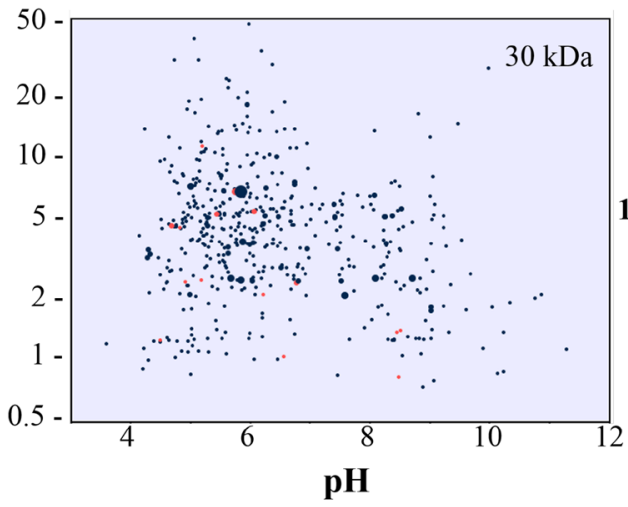

Fig. 1 a The chromatographic properties of CPB urine samples processed using 3, 10 or $30 \mathrm{kDa}$ MWCO filters in the modified-FASP method. The total ion chromatogram of each sample is given. Note the peak spreading and altered retention times in the 3 and $10 \mathrm{kDa}$ MWCO membrane processed samples. $\mathbf{b}$ The predicted molecular weight and pl distribution of proteins identified from the same urine sample FASP processed with 3 , 10 or $30 \mathrm{kDa}$ molecular weight cut-off membranes. The values beside each panel indicate the number of proteins with molecular weights less than $30 \mathrm{kDa}$ and the total number of proteins identified in sample

membranes may be used for sample preparation because of the efficiency of protein capture [25].

\section{Differential protein analysis}

Digests of the samples were analyzed by 2D-LC-MS and peptides were quantified by summing the intensities of all fragments in their associated CID spectra. Assignment of peptides into their source proteins was performed by $\mathrm{X}$ !tandem's algorithm, and we chose to use its primary protein assignments only. Protein level intensities were the sum of the intensities of member peptides, expressed a $\log _{2}$ scale for simple differential analysis.
High confidence protein identifications were based on detection of at least two distinct peptides each with $\log _{10}$ expectation scores of -1.5 or lower, roughly equivalent to a protein-level $\log _{10}$ expectation score of -3.0 or lower. This yielded the identification of 1154 proteins at the start of CPB and 915 proteins at 1-h into CPB surgery. The total number of unique proteins observed was 1325 , with 744 proteins identified in both samples (Additional file 2: Table S2). However, over a third of the population of the proteins identified (581) were seen exclusively at either the start of $\mathrm{CPB}$ or $1-\mathrm{h}$ into $\mathrm{CPB}$, rendering them ambiguous for quantitative analysis. This result highlights 
the challenge of comparative analysis of complex samples by mass spectrometry which arises as a result of the stochastic nature of instrument dependent data acquisition. Given the significant amount of time and effort committed to performing 2D DDA/IDA analysis and the variable complementarity of coverage between samples this was felt to be a suboptimal approach because such outcomes make it unclear if "missing" proteins in a sample were exclusively expressed in one set of the samples or if the peptides were simply not selected in one of the analyses. This motivated us to examine the feasibility of using a hyper reaction monitoring data independent approach [15].

As described in the Methods and Materials, we generated a large SWATH library by combining an existing human protein transition library [23] (henceforth called the "10K-library") and the results of our largerscale multiple 2D-LC-MS analysis of urines from cardiac bypass patients. Our approach was to replace our observed DDA/IDA transitions with their $10 \mathrm{~K}$-library counterparts for the peptides common to both library modes (and across their multiple charge state versions), and retain transition patterns for peptides observed in our samples' library but outside the 10K-library. The $10 \mathrm{~K}$-library's unitless iRT retention scale [26] was linearly mapped into retention time using a simple regression, exhibiting an excellent correlation of $\mathrm{R}^{2}>0.99$. The combined library contained 18,628 peptides spanning 3620 proteins; of these 3429 peptides spanning 934 proteins were not part of the original 10K-library but were detected in our 2D analysis of urine samples (Additional file 3: Table S3).

\section{SWATH analysis}

We applied the same two-peptide rule for SWATH quantitation as we did for the 2D DDA/IDA analysis, giving 4755 peptides spanning 730 proteins. This result suggested a remaining 1295 proteins were potentially quantifiable in SWATH, but by only a single peptide each. However, as quantitation based on a single peptide is less reliable these peptides were excluded from subsequent analysis. The complete peptide extraction report is available for additional exploration (see Availability of data and materials).

A total of 730 SWATH-quantifiable proteins were identified in the two sample pools, 552 of these also had quantitation values in both of the $2 \mathrm{D}$ LC runs. In addition there were 131 proteins that could be quantified using SWATH but not using the DDA/IDA derived data. These "gap filling" readouts indicated that SWATH presented the potential to differentially access approximately $22 \%$ of the proteins that the 2D IDA dataset saw as incomplete (Additional file 3: Table S3).

\section{Combined DDA/IDA and SWATH quantitation approaches}

The combined DDA/IDA and SWATH quantitation data were used to complement the quantitative analysis of each approach. The correlation between $\log _{2}$ SWATH and DDA/IDA protein expression values within a time point was moderate $\left(R^{2}=0.794\right.$ for start of $C P B$ across 651 common proteins; $\mathrm{R}^{2}=0.736$ for 1 -h into $\mathrm{CPB}$ across 584 common proteins), suggesting a general agreement between the readout technologies (Additional file 4: Table S4).

Applying a population filtering to these "acquisition technology-replicates" improved our quantitation reliability with a modest loss of total quantified proteins. By expressing the difference between the SWATH and DDA/IDA readouts (at the same time point) as normalized Z-scores, then discarding proteins with $|\mathrm{Z}|>1.65$ in either population, the resulting 475 common proteins ( $86 \%$ of the 552 protein initial list) improved the crosstechnology difference correlation to $\mathrm{R}^{2}=0.549$, while the correlation of difference values for the 77 proteins rejected by this filtering step is low $\left(R^{2}=0.01\right)$ (Fig. 2).

These results were encouraging considering that SWATH runs consumed approximately one-sixth of the instrument acquisition time, with one-third amount less sample preparation time as their 2D-DDA/IDA counterparts and fewer steps to potentially compromise quantitation. Going forward, the SWATH ion library will be applied to broader AKI studies for the analysis of individual patient urine samples, where the subsequent "large$\mathrm{N}$ " readout sets are amendable to more sophisticated statistical methods.

The supportive filtered population of 475 proteins formed the basis of our biological interpretation. In the absence of sampling statistics, we chose a fairly severe cut-off of threefold $\left(\log _{2} 1.59\right)$ protein expression difference required in both DDA/IDA and SWATH readouts. This gave 22 up-regulating proteins and 26 down-regulating proteins $1-\mathrm{h}$ into $\mathrm{CPB}$ versus the start of $\mathrm{CPB}$ (Table 2).

\section{Comparative analysis of the urinary proteome in patients undergoing $\mathrm{CPB}$}

The proteomic changes observed in these cardiac surgery patients were felt to reflect physiological responses to the trauma of the surgery and the associated bypass process, including ischemia reperfusion injury.

The majority of proteins that displayed altered expression after 1-h of CPB were localised to exosomes (Table 3). These findings are consistent with the observation that exosomes are derived from multivesicular bodies which fuse with renal tubular epithelial cell membranes before being secreted into the urine [27]. Urinary exosomes are typically isolated by differential 

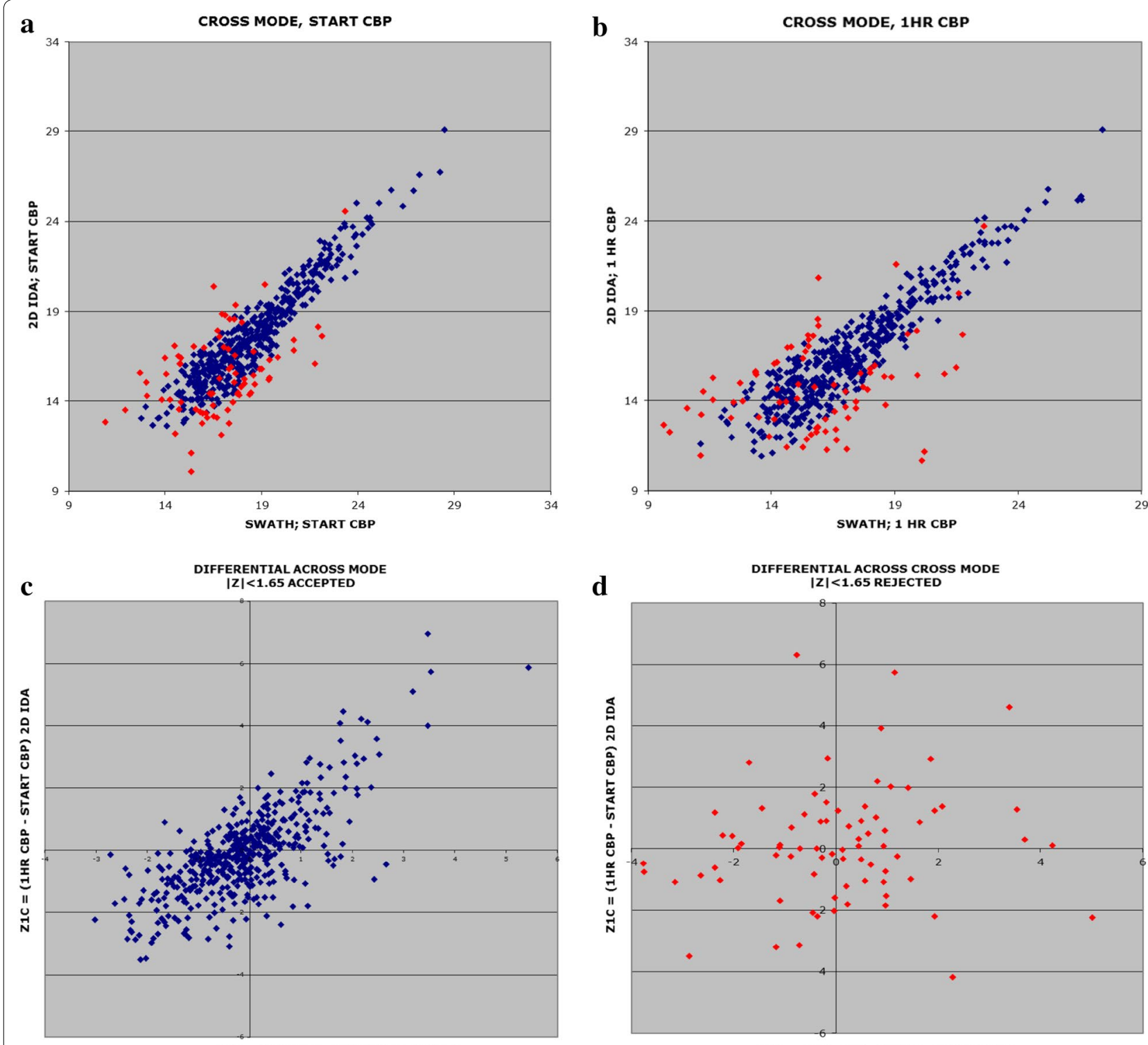

ZOC $=(1$ HR CBP - START CBP $)$ SWATH

Fig. 2 The use of protein level intensity correlations between 2D DDA/IDA and SWATH runs to improve quantitation reliability. Comparison of log 2 protein intensities $\mathbf{a}$ START of CPB $\mathbf{b}$ 1-hour CPB. c The expression of differences between SWATH and DDA/IDA readouts (at the same time point) as normalized Z-scores identifies proteins that show discordance between the two methods. This provides a basis for discarding the proteins with $|Z|>1.65$ in either population (as shown by the red dots), the resulting 475 common proteins ( $86 \%$ of the 552 protein initial list; the blue dots). This improved the cross-technology difference correlation to $R^{2}=0.549$. $\mathbf{d}$ The correlation of difference values for the 77 proteins rejected by this filtering step, shown is very low $\left(R^{2}=0.01\right)$

centrifugation prior to proteomic analysis, and identification of exosomal proteins here suggests that these methods may identify low abundance proteins; although proteome overlap between whole urine and exosomes has been reported [28]. These results could suggest that the repertoire of exosomal proteins changed during $\mathrm{CPB}$ but the process of exosome generation continued.
The up-regulated proteins were enriched for humoral immune responses (Table 4), which included predominantly innate features as outlined by the list of proteins involved (e.g. complement, lactotransferrin, myeloperoxisae and cathelicidin anti-microbial peptide). It is noteworthy that Chitinase-3-like protein 1 (CHI3L1, also called YKL-40) was also increased at 1-h post initiation 
Table 2 Correlation filtered 2D DDA/IDA and SWATH protein differences of magnitude greater than 1.59 (threefold up regulating at CPB-1-hour) from both methods marked in (A) and less than -1.59 (threefold down regulating at CPB-1hour) marked in (B)

\begin{tabular}{|c|c|c|c|}
\hline $\begin{array}{l}\text { SWATH } \\
\text { ( } \log _{2} \text { intensity ratio) }\end{array}$ & $\begin{array}{l}\text { DDA/IDA } \\
\text { ( } \log _{2} \text { intensity ratio) }\end{array}$ & UNIPROT entry & Description \\
\hline \multicolumn{4}{|l|}{ (A) } \\
\hline 2.53 & 3.09 & P01023 & Alpha-2-macroglobulin \\
\hline 1.78 & 3.53 & P04083 & Annexin A1 \\
\hline 2.05 & 3.04 & P04003 & C4b-binding protein alpha chain \\
\hline 3.53 & 5.73 & P00915 & Carbonic anhydrase 1 \\
\hline 1.82 & 4.47 & P49913 & Cathelicidin antimicrobial peptide \\
\hline 1.87 & 2.36 & P36222 & Chitinase-3-like protein 1 \\
\hline 3.47 & 4.00 & P08123 & Collagen alpha-2(I) chain \\
\hline 1.84 & 2.82 & P09871 & Complement $\mathrm{C} 1$ s subcomponent \\
\hline 1.86 & 2.01 & P02671 & Fibrinogen alpha chain \\
\hline 2.37 & 2.03 & P02675 & Fibrinogen beta chain \\
\hline 1.76 & 4.09 & P30043 & Flavin reductase (NADPH) \\
\hline 1.64 & 1.84 & P00738 & Haptoglobin \\
\hline 2.1 & 1.79 & P01860 & Ig gamma-3 chain $C$ region \\
\hline 2.09 & 1.99 & P13645 & Keratin, type I cytoskeletal 10 \\
\hline 2.3 & 4.12 & P02533 & Keratin, type I cytoskeletal 14 \\
\hline 3.48 & 6.95 & P08779 & Keratin, type I cytoskeletal 16 \\
\hline 5.44 & 5.87 & Q04695 & Keratin, type I cytoskeletal 17 \\
\hline 2.17 & 4.22 & P13647 & Keratin, type II cytoskeletal 5 \\
\hline 2.09 & 2.79 & P02788 & Lactotransferrin \\
\hline 3.18 & 5.10 & P14780 & Matrix metalloproteinase-9 \\
\hline 2.22 & 2.94 & P05164 & Myeloperoxidase \\
\hline 2.48 & 3.59 & P05109 & Protein S100-A8 \\
\hline \multicolumn{4}{|l|}{ (B) } \\
\hline-1.82 & -1.94 & P02763 & Alpha-1-acid glycoprotein 1 \\
\hline-2.35 & -2.10 & P05090 & Apolipoprotein D \\
\hline-2.14 & -3.50 & P55287 & Cadherin-11 \\
\hline-2.23 & -2.87 & P19022 & Cadherin-2 \\
\hline-1.64 & -1.80 & P49747 & Cartilage oligomeric matrix protein \\
\hline-2.39 & -2.86 & P39059 & Collagen alpha-1(XV) chain \\
\hline-1.66 & -2.32 & P08174 & Complement decay-accelerating factor \\
\hline-1.64 & -2.43 & Q9HCUO & Endosialin \\
\hline-1.92 & -2.97 & P17900 & Ganglioside GM2 activator \\
\hline-3.02 & -2.24 & Q96RW7 & Hemicentin-1 \\
\hline-2.31 & -2.30 & O75144 & ICOS ligand \\
\hline-2.31 & -2.63 & Q9BRK3 & Matrix-remodeling-associated protein 8 \\
\hline-1.72 & -2.20 & P08571 & Monocyte differentiation antigen CD14 \\
\hline-1.78 & -1.62 & Q9BXP8 & Pappalysin-2 \\
\hline-2.63 & -1.72 & P0DJD8 & Pepsin A-3 \\
\hline-2.16 & -1.72 & Q6UXB8 & Peptidase inhibitor 16 \\
\hline-1.7 & -2.24 & Q9HCN6 & Platelet glycoprotein VI \\
\hline-1.92 & -1.60 & P07602 & Prosaposin \\
\hline-1.88 & -2.83 & Q8WZ75 & Roundabout homolog 4 \\
\hline-1.79 & -2.70 & 000241 & Signal-regulatory protein beta-1 \\
\hline-2.02 & -3.47 & P19320 & Vascular cell adhesion protein 1 \\
\hline-2.16 & -2.73 & Q6EMK4 & Vasorin \\
\hline-1.79 & -2.34 & Q12907 & Vesicular integral-membrane protein VIP36 \\
\hline
\end{tabular}


Table 2 continued

\begin{tabular}{llll}
\hline $\begin{array}{l}\text { SWATH } \\
\left(\log _{\mathbf{2}} \text { intensity ratio) }\right.\end{array}$ & $\begin{array}{l}\text { DDA/IDA } \\
\left(\log _{\mathbf{2}} \text { intensity ratio) }\right.\end{array}$ & UNIPROT entry & Description \\
\hline-2.33 & -2.58 & Q96DA0 & Zymogen granule protein 16 homolog B \\
\hline
\end{tabular}

These formed the basis for our biological analysis

Table 3 GO cellular components enriched in differentially regulated proteins

\begin{tabular}{|c|c|c|c|c|}
\hline \multirow[t]{2}{*}{ Term } & \multicolumn{2}{|c|}{ Up regulated } & \multicolumn{2}{|c|}{ Down regulated } \\
\hline & Genes & $p$ value Bonferroni & Genes & $p$ value Bonferroni \\
\hline Extracellular exosome & 20 & $4.41 \times 10^{-14}$ & 23 & $4.99 \times 10^{-18}$ \\
\hline Extracellular membrane-bounded organelle & 20 & $4.41 \times 10^{-14}$ & 23 & $4.99 \times 10^{-18}$ \\
\hline Extracellular organelle & 20 & $4.79 \times 10^{-14}$ & 23 & $5.50 \times 10^{-18}$ \\
\hline Extracellular region part & 21 & $9.89 \times 10^{-14}$ & 22 & $3.22 \times 10^{-13}$ \\
\hline Membrane-bounded vesicle & 20 & $2.81 \times 10^{-12}$ & 23 & $6.18 \times 10^{-16}$ \\
\hline Extracellular region & 21 & $3.59 \times 10^{-12}$ & 22 & $1.33 \times 10^{-11}$ \\
\hline Vesicle & 20 & $5.11 \times 10^{-12}$ & 23 & $1.24 \times 10^{-15}$ \\
\hline Extracellular space & 15 & $1.82 \times 10^{-11}$ & 11 & $2.95 \times 10^{-5}$ \\
\hline Blood microparticle & 6 & $1.40 \times 10^{-6}$ & * & $*$ \\
\hline Cytoplasmic membrane-bounded vesicle lumen & 5 & $3.08 \times 10^{-5}$ & * & * \\
\hline Vesicle lumen & 5 & $3.27 \mathrm{E} \times 10^{-5}$ & * & * \\
\hline
\end{tabular}

Only cellular components enriched with a $p$ value of $10^{-5}$ or less are reported. Analysis was performed using String database [37]

of CPB. CHI3L1 is a member of the chilectin subfamily of the 18 glycosyl hydrolase family. This protein has retained the ability to bind heparin, collagen and chitin but it is enzymatically inactive [29]. It has been shown to have a number of immunostimulatory properties for the adaptive immune system as well as pro-inflammatory activities [29]. Increased levels of CHI3L1 have been associated with loss of renal function in diabetes, and renal or cardiac transplantation patients [30-33]. Our present observations suggest that CHI3L1 levels also increase in patients undergoing cardiac bypass surgery potentially as a result of intra operative subclinical ischemia-reperfusion injury.

These up-regulated proteins also contained a number of extracellular components, although these proteins appeared to derive more from vesicle or granule contents. ECM reorganization was a process characterized by the up-regulated proteins, including elevation of urinary MMP9. These findings are consistent with reports that demonstrate elevated urinary MMP7 and renal MMP2 expression in ischemia reperfusion injury of renal allografts $[4,34]$. While there was one diabetic patient in the cohort, it is unlikely that the acute intraoperative elevation of urinary MMP9 observed over 1-h of CPB was related to undocumented diabetic nephropathy [35].
Significantly, the up-regulated proteins also reflected general activation/regulation of innate immunity $(C 4 b$, lactotransferrin, protein S100-A8, myeloperoxidase) which is consistent with rapid, generic responses to cardiac surgery-induced tissue injury. Interestingly, there were also changes suggesting that the adaptive immune response might be down-regulated following 1-h on CPB, a process not previously described. Taken together, these findings corroborate pre-clinical models of the immune responses to renal ischemia reperfusion injury, but further expand on them to demonstrate that repair and re-modeling processes may be initiated sooner than previously thought [36].

It should be emphasised that the criteria used for the selection of proteins with significant changes between the two time points was based on the requirement that the protein was identified in both SWATH and DDA/ IDA analyses of the same sample, and the changes had to be of comparable magnitudes. These represent very stringent criteria to provide confidence regarding the presence and magnitude of the change using two independent analytical methods. This was not meant to imply that other quantitative changes predicted by observations made in only one of the approaches (DDA/IDA or SWATH) may not also be valid. However, the confidence 


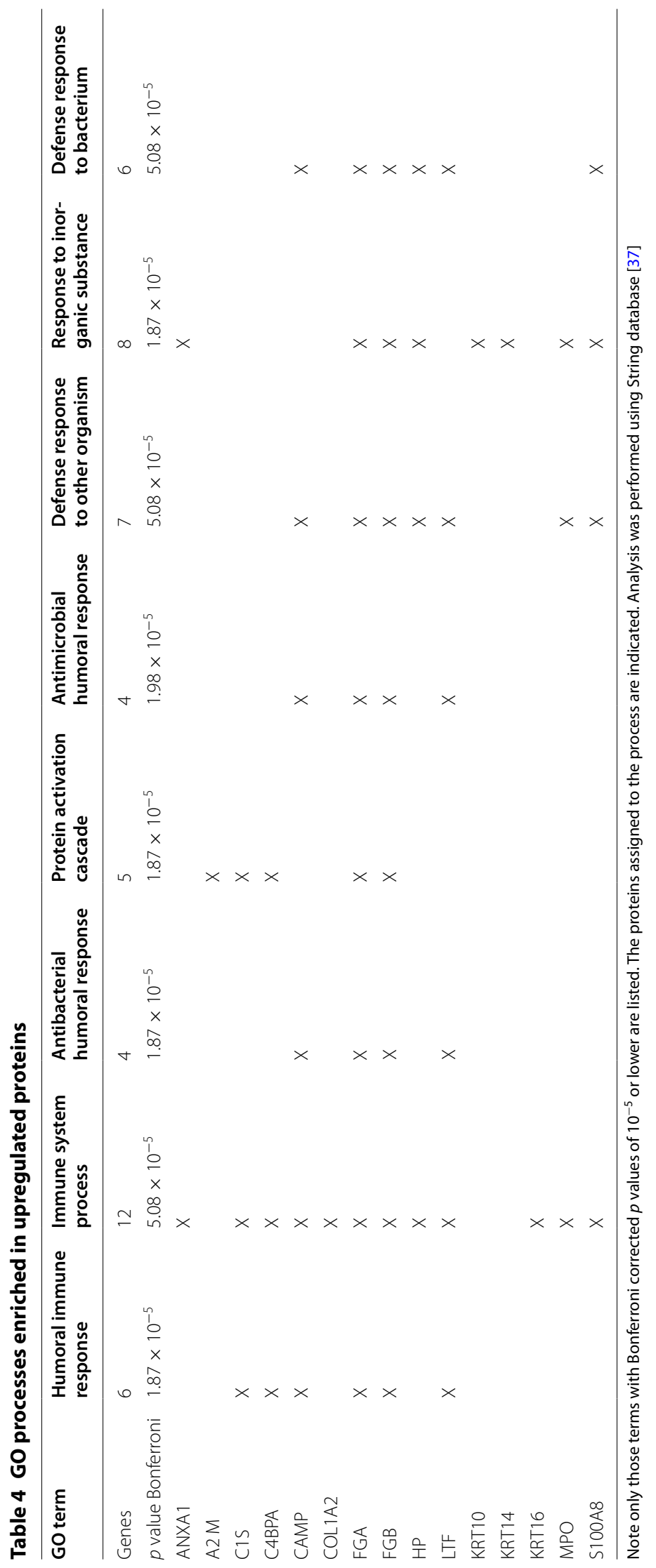


of such changes is lower and would require validation using alternate methodologies.

\section{Conclusions}

Our results provide a method for analyzing the urinary proteomic changes in adult patients undergoing cardiac surgery. Unlike the original FASP method the current procedure does not use SDS. The method requires limited sample processing to remove interfering substances and generates mass spectrometry ready samples. The design is such that it can be readily applied to large numbers of clinical samples with the potential for automation. The introduction of SWATH into the workflow offers several benefits to the analysis. The most significant aspect is the ability to consistently report on the same panel of $>700$ proteins in each analysis. This allows for more confident comparisons between samples, while keeping both the sample quantity requirements and the mass spectrometer instrument time relatively low. Although SWATH does not currently offer the analytical depth provided by 2D-LC-MS/ MS analyses, it may be possible to extend the level of quantitation through longer chromatographic run times or via pre-fractionation steps prior to SWATH-MS. There have been several comparisons to suggest that SWATH provides comparable quantitative analysis to other methods [22]. The approach outlined here is particularly relevant to the analysis of sequential urines from patients in various clinical conditions, including intraoperative samples.

\section{Additional files}

\begin{abstract}
Additional file 1: Table S1. The use of custom retention alignment peptides to map peptide retention times from 2D DDA/IDA runs into the SWATH extraction library, and their corresponding SWATH extracted retention times.

Additional file 2: Table S2. The log(2) protein values for DDA/IDA at time-points START and CPB-1-hour.

Additional file 3: Table S3. (A) An overview of the peptide and protein identifications and resulting differential quantitation for the 2D-LC DDA/ IDA and SWATH run datasets. (B) The impact of the application of a DDA/ IDA and SWATH intensity correlation filtering to the differential analysis.
\end{abstract}

Additional file 4: Table S4. Correlation filtered 2D DDA/IDA and SWATH protein difference values.

\begin{abstract}
Abbreviations
CPB: cardiopulmonary bypass; MS: mass-spectrometry; FASP: filter assisted sample preparation; SWATH: Sequential Window acquisition of All Theoretical fragment ion spectra; eGFR: estimated glomerular filtration rate; MWCO: molecular weight cut-off ultra-centrifugal filters; HPLC: high-performance liquid chromatography; 2D-LC-MS/MS: two dimensional liquid chromatography mass-spectrometry.
\end{abstract}

\section{Authors' contributions}

$\mathrm{RD}$ conducted the experiments, data analysis and helped in manuscript writing; MN helped conduct the experiments; NC collected the patient samples and completed the patient database; VS performed the bioinformatics and data processing; OK provided technical input on the mass-spectrometry and chromatography platform; CR and RA designed and implemented the prospective observational cohort of cardiopulmonary bypass patients; $\mathrm{JH}$ and JW were involved in the overall study design, data analysis, manuscript writing and obtaining funding. All authors read and approved the final manuscript.

\section{Author details}

${ }^{1}$ Manitoba Centre for Proteomics and Systems Biology, University of Manitoba and Health Sciences Centre, Room 799, John Buhler Research Center, 715 Mc Dermot Avenue, Winnipeg, MB R3E 3P4, Canada. ${ }^{2}$ Department of Internal Medicine, Section of Biomedical Proteomics, University of Manitoba, Winnipeg, MB, Canada. ${ }^{3}$ Department of Internal Medicine, Section of Nephrology, University of Manitoba, Winnipeg, MB, Canada. ${ }^{4}$ Department of Surgery, University of Manitoba, Winnipeg, MB, Canada. ${ }^{5}$ Cardiac Sciences Program, St. Boniface Hospital Research Centre, Winnipeg, Canada. ${ }^{6}$ Department of Immunology, University of Manitoba, Winnipeg, MB, Canada.

\section{Acknowledgements}

We would like to thank Peyman Ezzati for his role in method development and for his contributions to the sample analysis and mass spectrometry. The authors would like to gratefully acknowledge funding for this study from the Kidney Foundation of Manitoba, the Canadian Institutes of Health Research and Research Manitoba.

\section{Competing interests}

The authors declare that they have no competing interests.

\section{Availability of data and materials}

The supplementary data for this work is stored at the University of California, San Diego's MassIVE archive (massive.ucsd.edu) under index MSV000079693. The 2D DDA/IDA run's MGF files CPBSTART.mgf.gz and CPB1HR.mgf.gz are concatenated conversions from the raw instrument format, for the start of $C P B$ and 1-hour CPB time points, respectively. The SWATH peptide-level extraction report is stored under CPBSWATH-Peptides.txt, while CPB-system.txt contains an overall $\log _{2}$ protein expression matrix for both the 2D DDA/IDA and SWATH data.

\section{Ethics approval and consent to participate}

This study protocol was approved by the University of Manitoba health research ethics board (HS15221) and all patients provided informed consent.

\section{Funding}

This study was funded by support from the Kidney Foundation of Canada $(\mathrm{JH}, \mathrm{JAW})$, Canadian Institutes of Health Research (JH, JAW) and Research Manitoba $(J H)$. JH is supported by a CIHR New Investigator Salary Award. The funding body did not have any role in the study design, collection, analysis, data interpretation or writing of the manuscript.

Received: 2 May 2016 Accepted: 4 July 2016

Published online: 15 August 2016

\section{References}

1. Farrah T, Deutsch EW, Omenn GS, Sun Z, Watts JD, Yamamoto T, et al. State of the human proteome in 2013 as viewed through PeptideAtlas: comparing the kidney, urine, and plasma proteomes for the biology- and disease-driven Human Proteome Project. J Proteome Res. 2014;13(1):60-75

2. Aregger F, Pilop C, Uehlinger DE, Brunisholz R, Carrel TP, Frey FJ, et al. Urinary proteomics before and after extracorporeal circulation in patients with and without acute kidney injury. J Thorac Cardiovasc Surg. 2010;139(3):692-700

3. Ho J, Lucy M, Krokhin O, Hayglass K, Pascoe E, Darroch G, et al. Mass spectrometry-based proteomic analysis of urine in acute kidney injury following cardiopulmonary bypass: a nested case-control study. Am J Kidney Dis Off J Natl Kidney Found. 2009;53(4):584-95.

4. Ho J, Rush DN, Krokhin O, Antonovici M, Gao A, Bestland J, et al. Elevated urinary matrix metalloproteinase-7 detects underlying renal allograft inflammation and injury. Transplantation. 2016;100(3):648-54. 
5. Maddens B, Ghesquiere B, Vanholder R, Demon D, Vanmassenhove J, Gevaert K, et al. Chitinase-like proteins are candidate biomarkers for sepsis-induced acute kidney injury. Mol Cell Proteomics MCP. 2012;11(6):M111 013094.

6. Thongboonkerd V. Practical points in urinary proteomics. J Proteome Res. 2007;6(10):3881-90.

7. Loftheim H, Nguyen TD, Malerod H, Lundanes E, Asberg A, Reubsaet L. 2-D hydrophilic interaction liquid chromatography-RP separation in urinary proteomics-minimizing variability through improved downstream workflow compatibility. J Sep Sci. 2010;33(6-7):864-72.

8. Zhou H, Pisitkun T, Aponte A, Yuen PS, Hoffert JD, Yasuda H, et al. Exosomal Fetuin-A identified by proteomics: a novel urinary biomarker for detecting acute kidney injury. Kidney Int. 2006;70(10):1847-57.

9. Sigdel TK, Kaushal A, Gritsenko M, Norbeck AD, Qian WJ, Xiao W, et al. Shotgun proteomics identifies proteins specific for acute renal transplant rejection. Proteomics Clin Appl. 2010;4(1):32-47.

10. Sigdel TK, Salomonis N, Nicora CD, Ryu S, He J, Dinh V, et al. The identification of novel potential injury mechanisms and candidate biomarkers in renal allograft rejection by quantitative proteomics. Mol Cell Proteomics MCP. 2014;13(2):621-31.

11. Devarajan P, Krawczeski CD, Nguyen MT, Kathman T, Wang Z, Parikh CR. Proteomic identification of early biomarkers of acute kidney injury after cardiac surgery in children. Am J Kidney Dis Off J Natl Kidney Found. 2010;56(4):632-42.

12. Nguyen MT, Dent $C L$, Ross GF, Harris N, Manning PB, Mitsnefes MM, et al. Urinary aprotinin as a predictor of acute kidney injury after cardiac surgery in children receiving aprotinin therapy. Pediatr Nephrol. 2008;23(8):1317-26.

13. Adachi J, Kumar C, Zhang Y, Olsen JV, Mann M. The human urinary proteome contains more than 1500 proteins, including a large proportion of membrane proteins. Genome Biol. 2006;7(9):R80.

14. Nagaraj N, Mann M. Quantitative analysis of the intra- and interindividual variability of the normal urinary proteome. J Proteome Res. 2011;10(2):637-45.

15. Gillet $L C$, Navarro P, Tate $S$, Rost $H$, Selevsek N, Reiter L, et al. Targeted data extraction of the MS/MS spectra generated by data-independent acquisition: a new concept for consistent and accurate proteome analysis. Mol Cell Proteomics MCP. 2012;11(6):0111 016717

16. Guo T, Kouvonen P, Koh CC, Gillet LC, Wolski WE, Rost HL, et al. Rapid mass spectrometric conversion of tissue biopsy samples into permanent quantitative digital proteome maps. Nat Med. 2015;21(4):407-13.

17. Wisniewski JR, Zougman A, Nagaraj N, Mann M. Universal sample preparation method for proteome analysis. Nat Methods. 2009;6(5):359-62.

18. Dwivedi RC, Spicer V, Harder M, Antonovici M, Ens W, Standing KG, et al. Practical implementation of 2D HPLC scheme with accurate peptide retention prediction in both dimensions for high-throughput bottom-up proteomics. Anal Chem. 2008;80(18):7036-42.

19. Craig R, Cortens JP, Beavis RC. The use of proteotypic peptide libraries for protein identification. Rapid Commun Mass Spectrom RCM. 2005;19(13):1844-50.

20. Fenyo D, Beavis RC. A method for assessing the statistical significance of mass spectrometry-based protein identifications using general scoring schemes. Anal Chem. 2003;75(4):768-74.

21. Fenyo D, Eriksson J, Beavis R. Mass spectrometric protein identification using the global proteome machine. Methods Mol Biol. 2010;673:189-202.
22. McQueen P, Spicer V, Schellenberg J, Krokhin O, Sparling R, Levin D, et al. Whole cell, label free protein quantitation with data independent acquisition: quantitation at the MS2 level. Proteomics. 2015;15(1):16-24.

23. Rosenberger G, Koh CC, Guo T, Rost HL, Kouvonen P, Collins BC, et al. A repository of assays to quantify 10,000 human proteins by SWATH-MS. Sci Data. 2014;1:140031.

24. Krokhin OV, Spicer V. Peptide retention standards and hydrophobicity indexes in reversed-phase high-performance liquid chromatography of peptides. Anal Chem. 2009:81(22):9522-30.

25. Berger ST, Ahmed S, Muntel J, Cuevas Polo N, Bachur R, Kentsis A, et al. MStern blotting-high throughput polyvinylidene fluoride (PVDF) membrane-based proteomic sample preparation for 96-well plates. Mol Cell Proteomics MCP. 2015;14(10):2814-23.

26. Escher C, Reiter L, MacLean B, Ossola R, Herzog F, Chilton J, et al. Using iRT, a normalized retention time for more targeted measurement of peptides. Proteomics. 2012:12(8):1111-21.

27. Pisitkun T, Shen RF, Knepper MA. Identification and proteomic profiling of exosomes in human urine. Proc Natl Acad Sci USA. 2004;101(36):13368-73.

28. Salih M, Demmers JA, Bezstarosti K, Leonhard WN, Losekoot M, van Kooten $C$, et al. Proteomics of urinary vesicles links plakins and complement to polycystic kidney disease. J Am Soc Nephrol. 2016. [Epub ahead of print]

29. Di Rosa M, Distefano G, Zorena K, Malaguarnera L. Chitinases and immunity: ancestral molecules with new functions. Immunobiology. 2016;221(3):399-411.

30. De Loor J, Decruyenaere J, Demeyere K, Nuytinck L, Hoste EA, Meyer E. Urinary chitinase 3-like protein 1 for early diagnosis of acute kidney injury: a prospective cohort study in adult critically ill patients. Crit Care. 2016;20(1):38.

31. Malyszko J, Koc-Zorawska E, Malyszko J. YKL-40, a marker of cardiovascular disease and endothelial dysfunction, in kidney transplant recipients. Transplant Proc. 2014;46(8):2651-3.

32. Przybylowski P, Janik L, Wasilewski G, Nowak E, Kozlik P, Malyszko J. YKL-40, a novel marker of cardiovascular complications, is related to kidney function in heart transplant recipients. Transplant Proc. 2014;46(8):2860-3.

33. Zurawska-Plaksej E, Lugowska A, Hetmanczyk K, Knapik-Kordecka M, Adamiec R, Piwowar A. Proteins from the 18 glycosyl hydrolase family are associated with kidney dysfunction in patients with diabetes type 2 . Biomarkers. 2015;20(1):52-7.

34. Wanga S, Ceron CS, Delgado C, Joshi SK, Spaulding K, Walker JP, et al. Two distinct isoforms of matrix metalloproteinase- 2 are associated with human delayed kidney graft function. PLoS One. 2015;10(9):e0136276.

35. Li SY, Huang PH, Yang AH, Tarng DC, Yang WC, Lin CC, et al. Matrix metalloproteinase-9 deficiency attenuates diabetic nephropathy by modulation of podocyte functions and dedifferentiation. Kidney Int. 2014:86(2):358-69.

36. Rabb H, Griffin MD, McKay DB, Swaminathan S, Pickkers P, Rosner MH, et al. Inflammation in AKl: current understanding, key questions, and knowledge gaps. J Am Soc Nephrol JASN. 2016;27(2):371-9.

37. Jensen LJ, Kuhn M, Stark M, Chaffron S, Creevey C, Muller J, et al. STRING 8 - a global view on proteins and their functional interactions in 630 organisms. Nucleic Acids Res. 2009;37(Database issue):D412-6.

\section{Submit your next manuscript to BioMed Central and we will help you at every step:}

- We accept pre-submission inquiries

- Our selector tool helps you to find the most relevant journal

- We provide round the clock customer support

- Convenient online submission

- Thorough peer review

- Inclusion in PubMed and all major indexing services

- Maximum visibility for your research

Submit your manuscript at www.biomedcentral.com/submit 